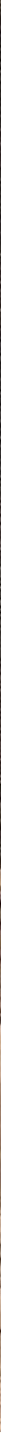

Karikatur zu einer Aufführung der Oper „Flavio" von Georg Friedrich Händel mit den Kastraten Senesino (1686-1757) (links) und Gaetano Berenstadt (1684-1734) (rechts) und der Sopranistin Francesca Cuzzoni (Mitte), Stich aus dem 18. Jahrhundert

\title{
"Engelsgleiche Stimmen"
}

\section{Sängerkastraten am Dresdner Hof}

\author{
Stephanie Hauptfleisch
}

Mit dem Aufkeimen des Kastratenwesens in der Kirchen- und Operngeschichte des 17. Jahrhunderts entstanden zahlreiche Mythen, Gerüchte und Spekulationen über die Sänger, die sich aufgrund ihrer operativ herbeigeführten physiologischen Sonderstellung einer stimmlichen wie auch körperlichen Geschlechterzuordnung entzogen und mit ihren hermaphroditischen Stimmen das Opernpublikum in ganz Europa begeisterten.

Die Akzeptanz von Sängerkastraten im gesellschaftlich-künstlerischen Leben stand zur damaligen Zeit nicht im offenen Widerspruch zu den moralischen Werten der Menschen, da es Frauen grundsätzlich nicht erlaubt war, in Kirchen zu singen. Die herrschenden Konventionen und Machtverhältnisse tolerierten so auf ignorante Weise Eingriffe in die intimen Bereiche der jungen Sänger, so dass es zur Akzeptanz und Förderung von Kastraten im Rahmen von Wissenschaft, Kunst und kirchlichen Institutionen kam. Auch wenn sich Kirche, Staat und Gesellschaft offiziell vom Ritus der Kastration distanzierten, wurden haltlose Gründe für die Notwendigkeit und Rechtfertigung dieser Operation ohne weiteres Hinterfragen hingenommen. Dass gerade die Kirche jahrzehntelang den größten Bedarf an Sängerkastraten hatte, entzog sich jeglicher Diskussion. Aufgrund der Verehrung und Begeisterung für diese außergewöhnlichen Stimmen wurde der Akt der körperlichen Verstümmelung öffentlich nie erörtert. Erst im Zuge der Aufklärung bildete sich mit der einhergehenden Veränderung 
des Kunstgeschmacks eine Motivationsgrundlage, die das selbstentblößende Wissen um die Priorität, die Dominanz und die unnatürlich herbeigeführte Körperlichkeit der Sängerkastraten einer allgemeinen Diskussion preisgab.

Eines der wichtigsten Betätigungsfelder der italienischen Kastratensänger im deutschsprachigen Raum war der Hof zu Dresden, der mit einer über Jahrhunderte gepflegten kulturellen Vielfalt das Leben in der sächsischen Residenzstadt maßgeblich bestimmte und als eine der herausragenden europäischen Musikpflegestätten galt. Über einen Zeitraum von fast 200 Jahren wirkten hier an die 80 Sängerkastraten, die unterschiedliche Anstellungsverhältnisse erlebten. In Abhängigkeit der herrschenden Kurfürsten und der eingesetzten Kapellmeister wurde die Italienische Oper am Haus gefördert oder beschränkt. So gab es für die Sängerkastraten, die ausschließlich aus Italien kamen, Zeiten fester Anstellung, höchster Gehälter und bester Bedingungen, aber auch schlechte Besoldung und befristete Anstellungsverhältnisse bei subventionierten Privattheatern.

Da nur eine geringe Anzahl der operierten Knaben tatsächlich ein Gesangsstudium abschließen und noch weniger stetige und große sängerische Erfolge vorweisen konnten, zeigten sich einige von ihnen als sehr vielseitig und weisen interessante und außergewöhnliche Biographien vor. Dazu zählt beispielsweise Giovanni Andrea Bontempi (1624-1705), der 1647 als erster Kastrat an den Dresdner Hof verpflichtet wurde. Er wurde 1657 zum Vizekapellmeister unter Heinrich Schütz (15851672) berufen und wirkte zudem als Architekt, Schriftsteller und Komponist. Von ihm stammt die erste vollständig erhaltene deutsche Oper mit dem Titel: „Musikalisch erhaltenes Schauspiel von der Dafne“, welche er gemeinsam mit dem Altisten und Kapellmeister Marco Giuseppe Peranda (1675-1675) komponiert hatte und welche $1672 \mathrm{im}$ neuerbauten Theater am Taschenbergpalais (Klengelsches Theater) vor Johann Georg II. (1613-1680) uraufgeführt wurde. Das Jahresgehalt von Bontempi und Peranda war dabei um einiges höher als das Einkommen von Oberkapellmeister Heinrich Schütz. ${ }^{2}$

Im sozialen Leben Dresdens erfuhren die Sänger zum Teil öffentlich hohe Anerkennung, doch wurden sie im privaten Umgang größtenteils gemieden und mit Vorurteilen betrachtet, wie ein Zitat von Gustav Schilling belegt: „Daß die Castraten von jeher und zu allen Zeiten in der Regel unsittliche Menschen waren, unterliegt keinem Zweifel, darf ihnen selbst aber we- niger zur Last gelegt werden.“3 Am sächsischen Hof genossen Kastraten zeitweise ein sehr hohes Ansehen. $\mathrm{Zu}$ nennen ist u. a. der Kastrat Bartolomäo Sorlisi (um 1632-1672), der 1662 an den Dresdner Hof kam und von Johann Georg II. sogar in den Adelsstand erhoben wurde. Ihm wurde zudem die Heirat mit einer Dresdnerin gestattet - ein Vorgang, der in der Geschichte des Kastratenwesens einmalig gewesen sein dürfte. Um die darauf empörte protestantische Bevölkerung zu beschwichtigen, ließ der Kastrat Sorlisi den Kirchturm zu Johnsbach erbauen. 1670 wurde er zum Amtmann von Dippoldiswalde erhoben.

Nur ein Jahr nach seiner Regierungsübernahme entließ Johann Georg III. (1647-1697) im Jahr 1680 alle italienischen Sänger, um eine „völlig deutsche Capell-Music“ zu gründen. ${ }^{4}$ Erst 1687 verpflichtete er wieder Kastraten an den Dresdner Hof, nachdem er 1685 mit Margherita Salicola (um 1682-1706) eine der ersten Primadonnen an den sächischen Hof gebracht hatte und nun die italienische Oper wiederbelebte.

Einen Höhepunkt am Dresdner Hof errreichte die italienische Oper zu den Hochzeitsfeierlichkeiten von Friedrich August II. (16961763). Sein Vater August der Starke (16701733) befürwortete das Ehevorhaben seines Sohnes, da dessen zukünftige Frau Maria Josepha erste Aspirantin des habsburgischen Erbes war. Da die italienische Opernpraxis am habsburgischen Kaiserhof höchst wertgeachtet wurde, scheute der sächsische Herrscher die horrenden Kosten für das Engagement der besten italienischen Sänger und Musiker nicht. Der europaweit bekannte Sopranist Francesco Bernardi, genannt Senesino (1686-1758), erhielt beispielsweise ein Jahresgehalt von 6.650 Taler, welches im Folgejahr auf den Rekordbetrag von 7.000 Talern erhöht wurde. Kapellmeister Antonio Lotti (1667-1740), der die Hochzeitsfeierlichkeiten musikalisch leitete, verdiente mit seiner Frau, der Sängerin Santa Stella Scarabini (1686-1759), zusammen 10.500 Taler jährlich, sodass allein diese drei Künstler den sächsischen Hof jährlich 17.500 Taler kosteten. Es sei darauf hingewiesen, dass der Kapelletat der gesamten Kapelle im Jahr 1697 bei ähnlichem Geldwert insgesamt 12.000 Taler betragen hatte. So ist es nicht verwunderlich, dass die Hochzeitsfeierlichkeiten zahlreiche Künstler und Musiker aus ganz Europa nach Dresden lockten. Auch Georg Friedrich Händel (1685-1759) kam in die Residenzstadt, um den genannten Senesino und weitere Sänger zu hören und nach London abzuwerben. Bereits 1720 verließen viele Sän-
2 Vgl. Stephanie Hauptfleisch: Sängerkastraten am Dresdner Hof, Magisterarbeit, TU Dresden 2007, S. 37.

3 Gustav Schilling: Encyclopädie der gesamten musikalischen Wissenschaften oder Universal-Lexikon der Tonkunst, Band II, Stuttgart 1842, S. 133.

4 Robert Prölss: Geschichte des Hoftheaters zu Dresden: von seinen Anfängen bis zum Jahre 1862, Dresden 1878, S. 102. 
Links: Der berühmte Kastrat Francesco Bernardi, genannt Senesino, welcher 1717 für ein Gehalt von 6650 Talern jährlich an den Dresdner Hof verpflichtet wurde. Stich nach Joseph Goupy (1689-1769)

Rechts: Der Kastrat Angelo Maria Monticelli, Gemälde von John Faber, um 1750

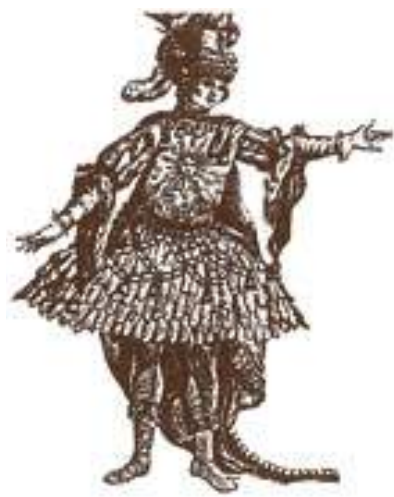

Der Kastrat Felice Salimbeni im Kostüm während einer Opernaufführung. Er wurde 1750 an den sächsischen Hof verpflichtet. Salimbeni wurde wegen fortdauernder Krankheit eine jährliche

Pension von 4.000 Talern zugestanden. Er verstarb im August 1751. Stich um 1745

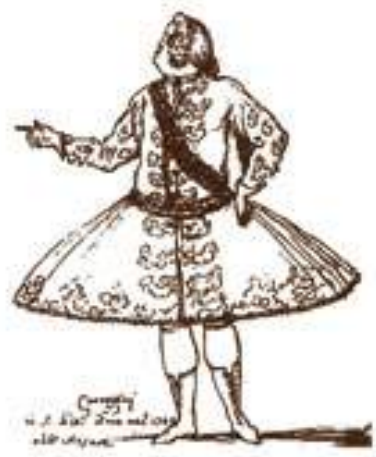

Giovanni Carestini, genannt Cusanino (1700-1760), der von 1746 bis 1750 am Dresdner Hof wirkte. Zeichnung von 1742

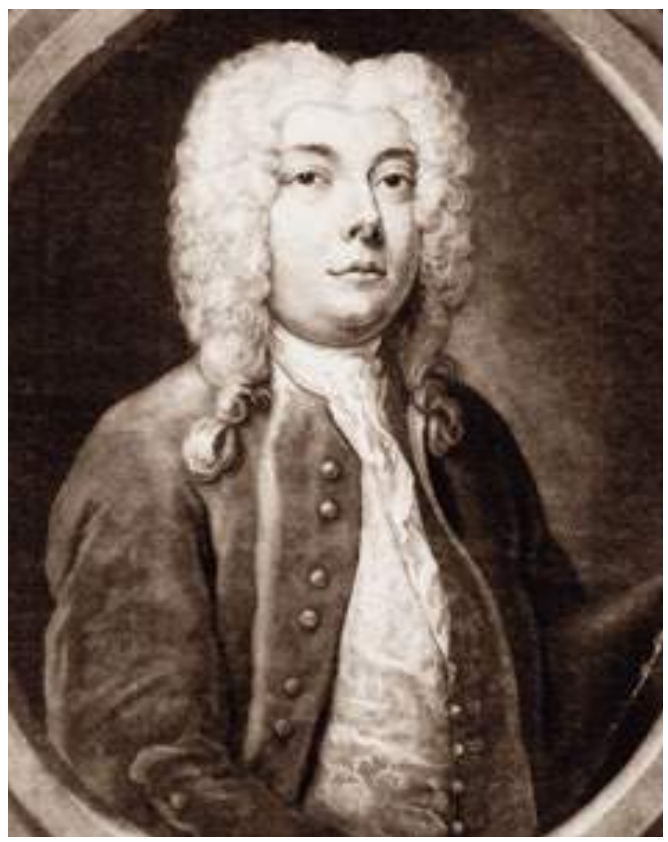

ger nach einem inszenierten Eklat den sächsischen Hof und folgten Händels Ruf nach London. Die den sächsischen Staatskassen zur Verfügung stehenden Gelder waren aufgebraucht.

Eine weitere Glanzzeit in der Geschichte der Dresdner Oper wurde durch Friedrich August II. (1696-1763) eingeleitet, der die italienische Oper am Dresdner Hof nach längerer Brachzeit wiederbeleben wollte. Er verpflichtete zahlreiche Musiker und Künstler an den sächsischen Hof. Mit dem Kapellmeister Johann Adolph Hasse (1699-1783) und seiner Frau, der Sängerin Faustina Bordoni (1675-1757), begann eine drei Jahrzehnte dauernde Ära, in der pompöse, barocke Opernvorstellungen und Festlichkeiten den Rahmen des höfischen Lebens bildeten. Die verpflichteten Kastraten, zu denen u. a. Felice Salimbeni (um 1712-1751), Salvator Pacifico, Giovanni Carestini, genannt Cusanino (1700-1760), Giovanni Bindi (gest. um 1750), Bartolomeo Putini, Angelo Maria Monticelli (1710-1758), Pasquale Bruscolini (1718-1782) und Giuseppe Perini gehörten, glänzten in den Hasseschen Werken. Der Siebenjährige Krieg beendete die ruhmreiche Ära Hasse und viele Musiker mussten den Dresdner Hof verlassen. Die Kastraten Pacifico und Perini blieben jedoch am sächsischen Hof, um die Ausgestaltung der Kirchenmusik zu gewährleisten. Perini betrieb ab 1794 ein Kaffee- und Tanzlokal am Altmarkt und gründete sogar eine eigene Theatergesellschaft unter seinem Namen („Perinische Gesellschaft"). ${ }^{5}$

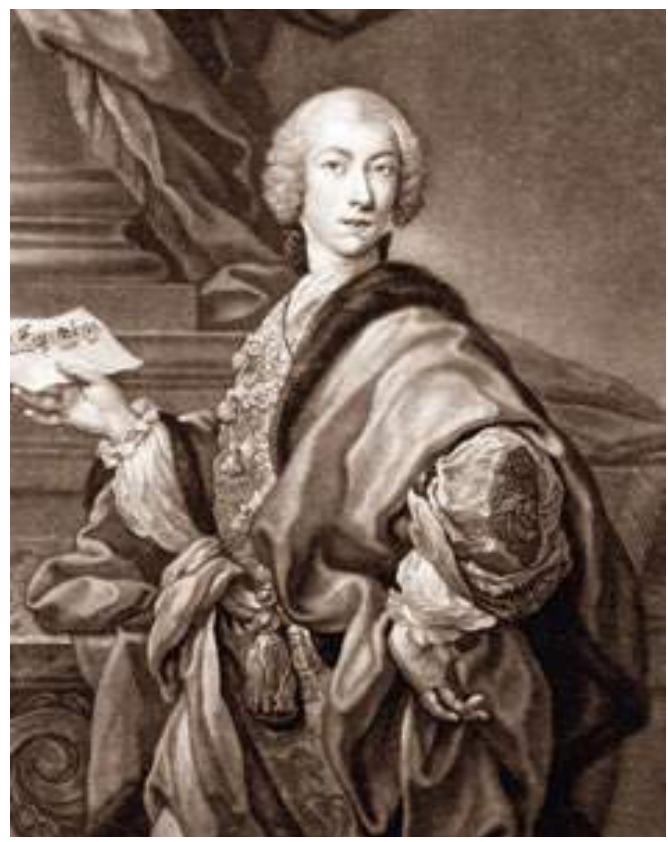

1765 wurde die italienische Oper als subventioniertes Privattheater unter Giuseppe Bustelli neu aufgestellt. In seiner Theatergesellschaft war u. a. der Sopranist Michele Patrassi, genannt Gibelli, beschäftigt. Bustelli kündigte allerdings 1778 aufgrund des Ausbruchs des Bayerischen Erbfolgekrieges. Sein Nachfolger, Antonio Bertoldi, brachte neue Kastratensänger nach Dresden, obwohl sich die Theaterwelt in Europa stark veränderte und Frauen nun gleichberechtigt mit Männern auf den Bühnen agierten. Zu einer Zeit, als Italien dem Kastratenwesen schon lange kritisch gegenüberstand, wurden in Dresden die letzten Kastratensänger gefeiert: Selbst noch Carl Maria von Weber (1786-1826) komponierte die Sopranpartie seine Missa Sancta in Es-Dur für den Sopranisten Felippo Sassaroli (1775-1828). In Dresden wurden die Sängerkastraten Anfang des 19. Jahrhunderts nunmehr hauptsächlich für die Kirchenmusik eingesetzt. Die Sänger Mosé Tarquini und Giovanni Muschetti waren die letzten Kastraten am Dresdner Hof. Eine Forderung Muschettis nach höherem Gehalt wurde allerdings abgelehnt und der Vertrag zu den bereits bestehenden Bedingungen 1841 verlängert. 1845 verließ Tarquini als letzter Sängerkastrat die sächsische Residenzstadt.

Erst im Jahr 1864 durften Sängerinnen als Solistinnen beim Kirchengesang eingesetzt werden. Bis dahin mussten die vorgetragenen Stücke an die Fähigkeiten der jugendlichen Sänger der Kapellknaben angepasst werden. Dies geschah unter der Anleitung 
des Kastraten Angelo Ciccarelli, einem Neffen Tarquinis, der als trauriges Zeugnis für ein mondänes Zeitalter der Musikgeschichte steht: Als letzter bediensteter Kastrat am Dresdner Hof widmete er sich ausschließlich der Lehrtätigkeit. Seinen Gesang hat man nie vernommen.

Die Faszination, welche der Gesang eines Kastraten auslöste, muss überwältigend gewesen sein: „Nur muß ich eines meisterhaften Künstlercoups erwähnen, durch den Sassaroli [Filippo Sassaroli] mich entzückte und überraschte. Er hielt nehmlich einen Ton ausnehmend lange aus, solange, daß mir die Brust zusammengeschnürt wurde, weil ich es unwillkürlich nicht wagte, Atem zu schöpfen, während der Sänger seinen Ton hielt. [...] Ich habe soetwas noch nicht gehört. Das geht in der That über alles Denken." ${ }^{6}$ Max Maria von Weber schrieb über Sassaroli: „Er war imstande den Ton kraftvoll 25 bis 30 Sekunden auszuhalten. [...] Das Timbre seiner Stimme hatte die Klangfarbe einer vollschwingenden, reinen Glasglocke und füllte die hallenden Räume der katholischen Kirche wie mit Engelsstimmen."7

Das Phänomen der lang gehaltenen Töne erklärt Franz Haböck: „Dieser Reichtum an Atem ist kein erworbener, sondern die natürliche Folge einer unnatürlichen, am Knaben verübten Operation.“ Haböck spricht hiermit ein Factum an, dass die Kastraten auch in optischer Hinsicht auffällig werden ließ: Aufgrund der vorpubertären Kastration wurde eine Verknöcherung der Wachstumsfugen verhindert, so dass die Betroffenen stetig weiterwuchsen, wohingegen sich die Stimmlippen nur geringfügig veränderten und kein auffälliger Stimmbruch stattfand. Hierdurch erreichten sie u. a. ein unglaubliches Lungenvolumen, aber auch ein auffälliges, voluminöses Äußeres, wie es auch von dem in Dresden angestellten und verstorbenen Kastraten Niccolo Pozzi in den „Dreßdnischen Merkwürdigkeiten“ berichtet wird: „Eodem ward der Königl. Kammermusikus, Herr Nikolaus Pozy, welcher etliche 50. Jahr alt am Brande gestorben, auf den Röm. Katholischen Begräbnisplatz nach Friedrichstadt unter einer zahlreichen Begleitung beygesetzet. Derselbe war unter dem Namen Nikolini allhier sehr bekannt, und ein ehemaliger berühmter Opernsänger, nachhero aber hat er nur in der Kirche sich hören lassen, weil er seines ausserordentlich fetten und korpulenten Leibes halber nicht das
Theater mehr betreten können. Er soll einige Center schwer gewogen haben, dahero auch sein Sarg in der Breite über zwei und eine halbe Elle ausgetragen, und nebst der Leiche nicht auf den ordentl. kathol. Leichenwagen, sondern auf einen so genannten Brancar oder Tragewagen abgeführet und fortgebracht werden müssen. Er hätte noch länger gelebet, wann er nicht durch ein Fußbad von Eisse, damit er einen Salzfluß am Schenkel zu vertreiben gedacht, seinen Tod verursachet." 88

Die Fettleibigkeit und die langen Glieder der Kastratensänger wurden in vielen zeitgenössischen Karikaturen dargestellt. Dass das äußere Erscheinungsbild natürlich eine wichtige Rolle spielte und den Erfolg eines Kastraten beeinflussen konnte, belegen die Schilderungen Metzlers, der beispielsweise über den Kastraten Ventura Rochetti (gest. 1755) schrieb: „Er ist ein langer wohlgewachsener Castrat. Sein Discant ist gut. Er singt schön, es ist wahr: nur zeigt er zu stark die elfenbeinenen Zähne. Man muß ja bei dem Singen den Mund eben nicht nothwendig so sehr auftun; denn sonst thäten es ja alle. Es ist dahero eine bloße Gewohnheit. Er hat sonst ein fein Gesicht, und recht schalkhafte Augen. Seine Blicke würden gefährlich seyn, wenn er schaden könnte." 9

Noch heute wird die Musik der damaligen Zeit mit Begeisterung aufgenommen und rezipiert. Dabei lösen die nun falsettierenden Interpreten, je höher sie in der Tonskala aufzusteigen in der Lage sind, zunehmende Verzückung, Euphorie und einen Begeisterungstaumel aus, der die Frage nach den Sängerkastraten der zurückliegenden Musikepochen vielleicht nicht umfassend stellt. Zuwenig wird und wurde diskutiert, unter welchem persönlichen Verzicht die Bewältigung der Musik durch die Sängerkastraten mit dem Anspruch zu erfüllen war, den die Kompositionen vorgaben. Zuwenig bedenkt und bedachte man, dass ein Eingriff in die individuelle Menschenwürde zur Grundlage einer bestimmten Musikrichtung wurde, dass diese Destruktion akzeptiert und wenn sie mit Erfolg bedacht war, ausgiebig gefeiert wurde. An den Rezipienten und Kunstliebhaber stellt sich somit die Aufgabe der kritischen Betrachtung und Hinterfragung der Bedingungen für die Musik. Die Brisanz um die Thematik des Kastratenwesens wird damit sicher noch keinen Abschluss finden.

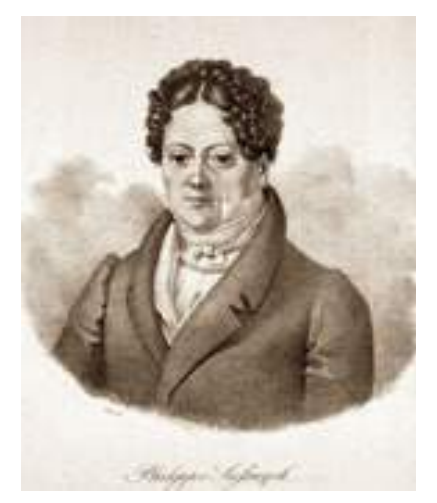

Filippo Sassaroli, einer der letzten Kastraten am Dresdner Hof. Stich um 1820
5 Vgl. Romy Petrick: Dresdens bürgerliches Musik- und Theaterleben im 18. Jahrhundert, Marburg 2010, S. $254 \mathrm{f}$.

6 Clemen Otto: Dresdner Eindrücke aus dem Jahre 1816 , in: Dresdner Geschichtsblätter, Nr. 1, 1918, S. 96.

7 Max Maria von Weber: Carl Maria von Weber. Ein Lebensbild, Band 1, Leipzig 1864, S. 171.

8 Peter Georg Mohrenthal: Kern Dreßdnischer Merkwürdigkeiten, Juni 1758, S. 41 f.

9 Johann Benedict Metzler: Beyträge zur Historie und Aufnahme des Theaters, Stuttgart 1750, S. 278. 